\title{
Problems on Bieberbach groups and flat manifolds
}

\author{
Andrzej Szczepański
}

February 2, 2008

In memory of Charles B. Thomas

\begin{abstract}
We present about twenty conjectures, problems and questions about flat manifolds. Many of them build the bridges between the flat world and representation theory of the finite groups, hyperbolic geometry and dynamical systems.
\end{abstract}

We shall present here some conjectures, problems and open questions related to flat manifolds. By flat manifold we understand a compact closed (without boundary) Riemannian manifold with sectional curvature equal to zero. It is well known (see for example 35]) that any flat manifold $M^{n}$ can be considered as an orbit space $\mathbb{R}^{n} / \Gamma$, where $\Gamma$ is a discrete, torsion free and cocompact subgroup of the group $E(n)=O(n) \ltimes \mathbb{R}^{n}=$ Isom $\mathbb{R}^{n}$. It is easy to see that $\Gamma=\pi_{1}\left(M^{n}\right)$. If we remove the assumption that $\Gamma$ is torsion free we obtain a crystallographic group. Hence we also consider the problems about them. From the Bieberbach theorems (see 4) any crystallographic group has a maximal free abelian subgroup (subgroup of all translations) of finite index. Hence for any such group $\Gamma \subset E(n)$ we have a short exact sequence

$$
0 \rightarrow \mathbb{Z}^{n} \rightarrow \Gamma \rightarrow H \rightarrow 0
$$

Here $H$ is a finite group which in case of torsion free crystallographic group (Bieberbach group) is isomorphic to the holonomy group of manifold $\mathbb{R}^{n} / \Gamma$. We call it the holonomy group of $\Gamma$. Since the subgroup $\mathbb{Z}^{n}$ is maximal abelian, 
the holonomy representation $\phi_{\Gamma}: H \rightarrow G L(n, \mathbb{Z})$, defined by conjugation in $\Gamma$, is faithful. We shall denote by $\alpha$ an element of the second cohomology group $H^{2}\left(H, \mathbb{Z}^{n}\right)$ which is defined by the above short exact sequence.

Finally we would like to mention that many conjectures, problems and open questions still make sense, if the context of flat manifolds (or equivalently Bieberbach groups) is replaced by the context of almost flat manifolds (or equivalently almost Bieberbach groups). Whenever this is the case, we shall denote this by $(*)$.

We do not pretend completeness of our list. In fact, we do not touch several questions about flat manifolds either because of our ignorance about their status or because of insufficient interest from our point of view.

We thank K.Dekimpe and Charles B. Thomas for their advice and help. We also would like to thank the referee for his (her) help and particulare for a correction of a few errors and improving the language.

\section{The classification problems}

It is well known (cf. 4]) that any finite group $G$ is a holonomy group of some flat manifold. Hence we have.

Problem $1{ }^{*}$ Find the minimal dimension of a flat manifold with the holonomy group $G$.

Remark 1 The answer is known for cyclic groups ([4], [11]), elementary abelian $p$ - groups, dihedral groups, semidihedral groups, generalized quaternion groups (see [13]) and simple groups $P S L(2, p)$ ( $p$ is a prime number) [23]. There are also some other classes of finite groups for which the minimal dimension is known. We do not pretend to give a complete list here. Unfortunately, for the most finite groups it seems to be very difficult question. For example, almost nothing is known for the symmetric groups. The main obstruction is the calculation of the second cohomology of the finite group with special coefficients.

Problem 2 Do the calculation for those p-groups for which the cohomology is well-understood. 
An inductive classification of the Bieberbach groups follows from E. Calabi (3], 35, Section 3.6 on page 124]). This is a consequence of the fact that any subgroup of the torsion free crystallographic group is again crystallographic. Hence any $n$-dimensional Bieberbach group $\Gamma$ with non trivial center is an extension of some Bieberbach group $\Gamma^{\prime}$ of dimension $n-1$ by integers $\mathbb{Z}$,

$$
0 \rightarrow \Gamma^{\prime} \rightarrow \Gamma \rightarrow \mathbb{Z} \rightarrow 0
$$

Hence we have the following problem.

Problem $3{ }^{*}$ For a finite group $G$ find a Bieberbach group (with trivial center) of minimal dimension.

Remark 2 The problem was considered in [12] (see also [13], [23]), where there are also some calculations. These include cyclic groups, elementary abelian p-groups (where $p$ is a prime number), dihedral 2-groups, semidihedral 2-groups, the generalized quaternion 2-groups and simple groups of Lie type.

In 1972 A. T. Vasquez [33] introduced, for any finite group $G$, an invariant $n(G) \in \mathbb{N}$. This is related to the class of flat manifolds with holonomy group $G$. In 1989 in the beautiful paper [5] G. Cliff and A. Weiss, calculated $n(G)$ for any finite $p$-group. A purely algebraic definition of $n(G)$ is given in [29]. There is also given a characterization of the groups with $n(G)=1$.

Problem 4 Calculate Vasquez invariant $n(G)$ for a finite group $G$.

Remark 3 There is a method, complementary to that of Calabi for the classification of Bieberbach groups with given holonomy group $G$. It says that any Bieberbach group $\Gamma$ with holonomy group $G$ and dimension $n \geq n(G)$ can be defined by a short exact sequence of groups

$$
0 \rightarrow \mathbb{Z}^{n-n(G)} \rightarrow \Gamma \rightarrow \Gamma_{G} \rightarrow 0
$$

where $\Gamma_{G}$ is a Bieberbach group of dimension $n(G)$, (cf. [29]).

Let $f: M^{n} \rightarrow M^{n}$ be a continuous map, and let $\tilde{f}: \mathbb{R}^{n} \rightarrow \mathbb{R}^{n}$ be its cover in the Euclidean space. From the third Bieberbach theorem (see [35]), we have the following short exact sequence (cf. 4])

$$
0 \rightarrow \operatorname{Aff} f_{0}\left(M^{n}\right) \rightarrow \operatorname{Aff}\left(M^{n}\right) \rightarrow \operatorname{Out}(\Gamma) \rightarrow 0
$$


where

$$
\text { Aff }\left(M^{n}\right)=\left\{f: M^{n} \rightarrow M^{n} \mid \tilde{f}: \mathbb{R}^{n} \rightarrow \mathbb{R}^{n} \in G L(n, \mathbb{R}) \ltimes \mathbb{R}^{n}\right\} .
$$

Af $f_{0}\left(M^{n}\right)$ denotes the connected component of the identity of the group of affine diffeomorphisms of the flat manifold $M^{n}$. It is isomorphic (see [4]) to $\left(S^{1}\right)^{\beta_{1}\left(M^{n}\right)}$, where $\beta_{1}$ denotes the first Betti number of the manifold, see [12]. Moreover we have the following a short exact sequence (see [15] and [4])

$$
0 \rightarrow H^{1}\left(H, \mathbb{Z}^{n}\right) \rightarrow O u t(\Gamma) \rightarrow N_{\alpha} / H \rightarrow 0,
$$

where $N=N_{G L(n, \mathbb{Z})}(H)$ is the normalizer of the holonomy group $H$ in $G L(n, \mathbb{Z})$ and

$$
N_{\alpha}=\{n \in N \mid n * \alpha=\alpha\} .
$$

Here $*$ is the standard action of the normalizer in cohomology, see 4, page 168]. Hence we have.

Theorem 1 ([31]) The following conditions are equivalent:

(i) $\operatorname{Out}(\Gamma)$ is a finite group,

(ii) the normalizer $N$ is a finite group,

(iii) the holonomy representation $\phi_{\Gamma}$ is $\mathbb{Q}$-multiplicity free and any

$\mathbb{Q}$-irreducible component is also $\mathbb{R}$-irreducible.

Proof: The equivalence of the conditions $(i)$ and $(i i)$ is obvious. The proof of the last equivalence is much more difficult and we refer the reader to [31].

We can now fomulate the following,

Problem $5 *$ Classify finite groups which are holonomy groups of flat manifolds $M^{n}=\mathbb{R}^{n} / \Gamma$ with finite outer automorphism group Out $\Gamma$.

Remark 4 Some progress was made in [15], e.g. for finite abelian groups.

From a finite group representation theory point of view, it might be interesting to consider a similar classification for Kähler flat manifolds (see [27]). Note that, here $\operatorname{Out}(\Gamma)$ is finite if and only if the holonomy representation is $\mathbb{Q}$-multiplicity free and any $\mathbb{R}$-irreducible component is $\mathbb{C}$-reducible.

The next conjecture is related to the last problem. 
Conjecture 1 ([28]) For any finite group $H$, there exist a Bieberbach group $\Gamma$ with $\mathbb{Q}$ multiplicity free holonomy representation $\phi_{\Gamma}$.

Remark 5 If the first Betti number of the manifold $M^{n}$ is zero then, from above, we have

$$
\text { Aff }\left(M^{n}\right) \simeq \operatorname{Out}(\Gamma) .
$$

This means that all information about the symmetries of the manifild is built into the outer automorphism group.

It is interesting to compare the last problem with the work of M. Belolipetsky and A. Lubotzky [2] where they proved that for any finite group $G$ there exist a fundamental group $\Gamma$ of some compact hyperbolic manifold such that $\operatorname{Out}(\Gamma)=G$. Hence we have.

Problem 6 Which finite groups $G$ occur as outer automorphism groups of Bieberbach groups with trivial center.

In this connection we can ask if every finite subgroup of $G L(n, \mathbb{Z})$ has a realisation as the centraliser of some finite subgroup of $G L(n, \mathbb{Z})$, for some $n$.

Remark 6 In [34] there is an example of a flat manifold whose fundamental group has trivial center and trivial outer automorphism group.

\section{The Anosov relation for flat manifolds and Entropy Conjecture}

Let $f: M \rightarrow M$ be a continuous map of a smooth manifold $M$. In fixed point theory, there exist two numbers associated to $f$ which provide some information on the fixed point set of $f$ : the Lefschetz number $L(f)$ and the Nielsen number $N(f)$. It is known that the Nielsen number provides more information about the fixed point set than $L(f)$ does, but $L(f)$ is easier to calculate. For nilmanifolds however, D. Anosov [1] showed that $N(f)=|L(f)|$ for any self map $f$ of the nilmanifold. On the other hand, he also observed that this result could not be extended to the class of all infranilmanifolds, since he was already able to construct a counter-example on the Klein Bottle. It was recently shown that for large families of flat 
manifolds (e.g. all flat manifolds with an odd order holonomy group) the Anosov relation $N(f)=|L(f)|$ does hold for any self map (see [6] and [7]). It is therefore natural to ask.

Problem $7^{*}$ (K. Dekimpe) Describe the class of flat manifolds (or more generally the class of infra-nilmanifolds) on which the Anosov relation

$$
N(f)=|L(f)|
$$

does hold for any map $f: M \rightarrow M$.

One can also consider this problem from a different point of view and fix the flat manifold $M$ and try to find all self maps $f$ for which the Anosov relation is valid.

Problem $8^{*}$ (K. Dekimpe) Given a flat manifold $M$. Is it possible to determine classes of self maps $f: M \rightarrow M$ for which the Anosov relation

$$
N(f)=|L(f)|
$$

does hold?

A first result in this direction can be found in [19].

Let $n \in \mathbb{Z}^{+}$and $\epsilon \in \mathbb{R}^{>0}$. Moreover let $(X, d)$ be a compact metric space and $f: X \rightarrow X$ a continuous map. Put

$$
r(f, \epsilon):=\lim _{n \rightarrow \infty} \sup \frac{1}{n} \log \max \{\# Q: Q \subset X\},
$$

where $Q$ is such that for any distinct points $x, y \in \mathbb{Q}$

$$
\max _{0 \leq j \leq n-1} d\left(f^{j}(x), f^{j}(y)\right) \geq \epsilon .
$$

The topological entropy $h(f)$ is a nonnegative real number or $\infty$ defined

$$
h(f)=\lim _{\epsilon \rightarrow 0} r(f, \epsilon)=\sup _{\epsilon \rightarrow 0} r(f, \epsilon) .
$$

Now assume that $X$ is a compact smooth manifold $M$ of dimension $m$. Let

$$
H^{*}(f): H^{*}(M, \mathbb{R}) \rightarrow H^{*}(M, \mathbb{R})
$$

be the linear map induced by $f$ on the cohomology space $H^{*}(M, \mathbb{R}):=$ $\oplus_{i=0}^{m} H^{i}(M, \mathbb{R})$ of $M$ with real coefficients. By $s p(f)$ we denote the spectral radius of $H^{*}(f)$, which is a homotopy invariant. In 1974 M.Shub asked in [26, page 36] the extent to which the following inequality holds. 
(EC) $\quad \log \operatorname{sp}(f) \leq h(f)$.

Then A. Katok in [16] put the following Entropy Conjecture.

Conjecture 2 (EC) holds for all continuous mappings $f: M \rightarrow M$, if $M$ is a manifold with the universal cover homeomorphic to $\mathbb{R}^{n}$.

It was proved for $M$ being tori and nilmanifolds, see [20].

Problem $9\left(^{*}\right)$ Give a proof of the (EC) for flat manifolds.

For any flat manifold $M$ with the first Betti number equal to zero and finite outer automorphism group of the fundamental group $\pi_{1}(M)$ the above conjecture is true. In fact, (see remark 5 and theorem 1) for any continuous map $f: M \rightarrow M$ the induced map $H^{*}(f)$ has a finite order.

We send the reader to [20] for more informations about the Entropy Conjecture*.

\section{Generalized Hantzsche-Wendt flat manifolds}

Let us agree to call any fundamental group of the $n$-dimensional flat manifold with holonomy group $\left(\mathbb{Z}_{2}\right)^{n-1}$ a generalized Hantzsche-Wendt $(G H W)$ Bieberbach group (see 24]). If the manifold is orientable, we call the $G H W$ Bieberbach group orientable. For dimension 3 there is only one oriented flat manifold $M^{3}$ with holonomy group $\mathbb{Z}_{2} \oplus \mathbb{Z}_{2}$. It was first considered by Hantzsche and Wendt. From the other side the fundamental group $\pi_{1}\left(M^{3}\right)$ is group $F(2,6)$, where $F(r, n)$ is the group defined by the presentation

$$
\begin{gathered}
<a_{1}, a_{2}, \ldots, a_{n} \mid a_{1} a_{2} \cdots a_{r}=a_{r+1}, a_{2} a_{3} \cdots a_{r+1}=a_{r+2}, \ldots, \\
a_{n-1} a_{n} a_{1} \cdots a_{r-2}=a_{r-1}, a_{n} a_{1} a_{2} \cdots a_{r-1}=a_{r}>,
\end{gathered}
$$

where $r>0, n>0$, and all subscripts are assumed to be reduced modulo $n$. This group is named a Fibonacci group.

Problem 10 Explain the relation between orientable GHW Bieberbach groups and the Fibonacci groups.

$*$

We would like to thank W. Marzantowicz for putting our attention on this problem. 
Remark 7 For any even natural number $2 n$ there exists an epimorphism

$$
\Phi_{2 n}: F(2 n, 2(2 n+1)) \rightarrow \Gamma_{2 n},
$$

where $\Gamma_{2 n} \subset E(2 n+1)$ denote an orientable $G H W$ Bieberbach group of rank $(2 n+1)$, see [30]. Nothing is known about the kernel of the epimorphism $\Phi_{2 n}$, for $n>1$.

Problem 11 Give definition of any GHW Bieberbach group in terms of generators and relations.

Let $\Gamma$ be a GHW Bieberbach group of dimension $n$ and with trivial center. It is well known ([10, Theorem 1.1, page 183], 14]) that there is an epimorphism of $\Gamma$ onto the infinite dihedral group $\mathbb{Z}_{2} * \mathbb{Z}_{2}$. Hence we have a decomposition

$$
\text { (*) } \quad \Gamma \simeq \Gamma_{1} *_{X} \Gamma_{2},
$$

as the generalized free product of two Bieberbach groups of dimension $(n-$ 1), amalgamated over a subgroup $X$ of index two. Moreover any GHW Bieberbach group, with non-trivial center, is the semidirect product of $\mathbb{Z}$ with some lower dimensional GHW Bieberbach group. We have.

Conjecture 3 For any GHW Bieberbach group $\Gamma$ with trivial center and dimension $n$, there exists a decomposition $(*)$ such that $\Gamma_{1}$ and $\Gamma_{2}$ are $G H W$ Bieberbach groups of dimension $n-1$.

Remark 8 The conjecture is true for $n \leq 4$ (see [14, page 30 and 38]).

For the remaining problem we have:

let $M^{n}$ be a flat oriented manifold with GHW fundamental Bieberbach group and dimension $n$. It is well known, see [24, that $M^{n}$ is a rational homology sphere.

Problem 12 What are the topological (geometrical) properties of $M^{n}$ ? 


\section{Flat manifolds and other geometries}

Let us recall the following well known question.

Question (Farrell-Zdravkovska) 9]) Let $M^{n}$ be a $n$-dimensional flat manifold. Is there a $(n+1)$-dimensional hyperbolic manifold $W$ the boundary of which equals $M^{n}$ ?

We would like to mention that the above question is very close related to the following one. Let $V^{n+1}$ be a hyperbolic Riemannian manifold (constant negative curvature) of finite volume. It is well known that $V^{n+1}$ has finite number of cusps and each cusp is topologicaly $M^{n} \times \mathbb{R}^{\geq 0}$, where $M^{n}$ is $n$ dimensional flat manifold. Is there some $V^{n+1}$ with one cusp homeomorphic to $M^{n} \times \mathbb{R}^{\geq 0}$ ?

D.D.Long and A.Reid proved ([17]) that the answer to the problem above is negative if the $\eta$-invariant of the signature operator of $M^{n}$ is not an integer. To prove it they used the Atiyach, Patodi, Singer formula, where signature operator $D$ means some differential elliptic operator and its $\eta$-invariant measure the symmetry of the spectrum of $D$. They also proved that there exists, already in dimension three, a flat manifold which signature operator has the $\eta$-invariant $\notin \mathbb{Z}$. By our assumptions this method works only in dimension $4 k-1$.

Problem 13 Classify flat manifolds with non-integral signature $\eta$-invariant.

They also proved ([18]) that any flat manifold has a "realization" as a cusp of some hyperbolic orbifold of finite volume.

Remark 9 We have the following flat manifolds of dimension two: the torus and the Klein bottle. The first one has a "realization" as a cusp of the eight knot complement and the second one as a cusp of the Gieseking manifold, (c.f. [32]). In dimension three we have ten flat manifolds and each one has "realization" as a cusp of some four dimensional hyperbolic manifold $W$, which is not necessarly one-cusped, (see [22]).

One major difficulty is the lack of examples of hyperbolic manifolds. In particular we have.

Problem 14 Give an example of a four dimensional hyperbolic manifold of finite volume with only one cusp. 
The $\eta$-invariant of the signature operator for a flat manifold is an important quantity as we have observed above. Let us ask the same question about the Dirac operator. We shall need our orientable flat manifold $M^{n}$ to have a spin structure. It turns out that this is equivalent to the existence of a homomorphism $\epsilon: \Gamma \rightarrow \operatorname{Spin}(n)$ such that the following diagram is commutative

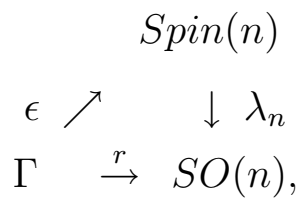

where $\lambda_{n}$ is the universal covering and $r$ is the projection onto the linear part, [8].

Problem $15 *$ Classify the holonomy groups of flat manifolds which admit a spin structure

Remark 10 It is known [8, Proposition 1, Corollary 1] that any flat manifold with holonomy group of odd order or of order $2 k, k$ an odd number, has a spin structure. However, see [21, Theorem 3.2], for the holonomy group $\mathbb{Z}_{2} \times \mathbb{Z}_{2}$, there exist orientable flat manifolds $M_{1}, M_{2}$ of dimension 6 with different holonomy representations $h_{1}, h_{2}: \mathbb{Z}_{2} \times \mathbb{Z}_{2} \rightarrow G L(6, \mathbb{Z})$, only one of which has a spin structure.

Question Is there an example of an oriented flat spin - manifold for which the Dirac $\eta$-invariant is not equal to the signature $\eta$-invariant modulo $\mathbb{Z}$ ?

For the methods of calculation of the Dirac $\eta$-invariant see [25].

A six dimensional flat manifold is Calabi-Yau if and only if its holonomy representation has the property that each $\mathbb{R}$-irreducible summand, which is also $\mathbb{C}$-irreducible, occurs with even multiplicity. Hence the classification of such manifolds is possible, see [27.

Problem 16 What are the properties of the Calabi-Yau flat manifolds. Do they have some "mirror-symmetry"?

\section{Acknowledgements}

This work was carried out in the framework of the project BIL01/C-31 for Bilateral Scientific Cooperation (Flanders-Poland) and was supported by University of Gdańsk BW - 5100-5-0149-4. 


\section{References}

[1] D. Anosov, The Nielsen numbers of maps of nil-manifolds. Uspekhi Mat. Nauk., 40, (1985), 133-134. English transl.: Russian Math. Surveys, 40, (1985), 149-150

[2] M. Belolipetsky, A. Lubotzky, Finite groups and hyperbolic manifolds, Invent. Math. 162 (3), 2005, 459 - 472

[3] E.Calabi, Closed locally euclidean four dimensional manifolds. Bull.A.M.S. 63, (1957) 135

[4] L. S. Charlap, Bieberbach Groups and Flat Manifolds, Springer-Verlag, 1986

[5] G. Cliff, A. Weiss, Torsion free space groups and permutation lattices for finite groups, Contemp. Math. 93 (1989), 123-132. (1977), 182-203

[6] K. Dekimpe, B. De Rock, W. Malfait, The Anosov theorem for infranilmanifolds with cyclic holonomy group. Preprint, 2004

[7] K. Dekimpe, B. De Rock, H. Pouseele, The Anosov relation for Nielsen numbers of maps of infra-nilmanifolds. Preprint, 2005

[8] K. Dekimpe, M. Sadowski, A. Szczepański; Spin structures on flat manifolds, to appear in Monatshefte für Mathem.

[9] F.T.Farrell, S.Zdravkovska, Do almost flat manifolds bound ?, Michigan J.Math. 30, (1983), 199-208

[10] F.T.Farrell, W.C.Hsiang, The topological Euclidean space form problem, Invent.Math. 48, (1978), 181-192

[11] H. Hiller, Bieberbach groups with cyclic holonomy groups, preprint

[12] H. Hiller, C. H. Sah; Holonomy of flat manifolds with $b_{1}=0$, Quartely J. Math. 37 (1986), 177-187

[13] H. Hiller, Z. Marciniak, C. H. Sah, A. Szczepanski, Holonomy of flat manifolds with $b_{1}=0$ II. Q. J. Math. 38 (1987), 213-200;

[14] J. Hillmann, Flat 4-manifold groups, New Zealand J.Math. 24, (1995), 29-40. 
[15] G.Hiss, A.Szczepański, Holonomy groups of Bieberbach groups with finite outer automorphism groups. Arch.Math., 65, (1995) 8-14.

[16] A. W. Katok, Entropy conjecture, Smooth Dynamical Systems, Mir Publishing, Moskow

[17] D.D.Long, A. W. Reid; On the geometric boundaries of hyperbolic 4manifolds. Geom. Topol. 4 (2000), 171-178;

[18] D.D.Long, A.W.Reid, All flat manifolds are cusps of hyperbolic orbifolds, Algebraic and Geometric Topology, Vol. 2 (2002), 285-296

[19] W. Malfait, The Nielsen numbers of virtually unipotent maps on infranilmanifolds. Forum Math., 13, (2001), 227-237

[20] W. Marzantowicz, F. Przytycki, Entropy conjecture for continuous maps on nilmanifolds, preprint , 2005, Poznań

[21] R.J. Miatello, R. A. Podestá, Spin structures and spectra of $\mathbb{Z}_{2}^{k}$ manifolds, Math. Zeitschrift. 247 (2004), 319-335

[22] B.E.Niemershein, All flat three-manifolds appear as cusps of hyperbolic four manifolds, Topology and Its Appl. 90, (1998), 109-133

[23] W. Plesken Minimal dimensions for flat manifolds with prescribed holonomy. Math.Ann. 284, (1989) 477-486

[24] J.P. Rossetti, A,Szczepański, Generalized Hantzsche-Wendt flat manifolds, Revista Matem. Iberoamer. 21, (2005) No. 3, 1053 - 1070

[25] M.Sadowski, A.Szczepański, Flat manifolds, harmonic spinors, and eta invariants, to appear in Adv. in Geometry

[26] M. Shub, Dynamical systems, filtrations and entropy, Bulletin AMS, 80, No.1, (1974), 27-41

[27] A.Szczepański, Kähler flat manifolds of low dimensions, preprint, $\mathrm{M} / 05 / 43$ - IHES

[28] A.Szczepański, Holonomy groups of crystallographic groups with finite outer automorphism groups, Group Theory and Low Dimensional Topology, German-Korea Workshop, Pusan 2000, (J.Mennicke, Jung Rae Cho 
(eds.)) in Research and Exposition in Mathematics, Vol. 27 (2003), 163166

[29] A. Szczepański, Decomposition of flat manifolds, Mathematika, 44 (1997), 113-119

[30] A. Szczepański, The euclidean representations of the Fibonacci groups, Q. J. Math. 52 (2001), 167-180;

[31] A. Szczepański, Outer automorphism groups of Bieberbach groups. Bull.Belg.Math.Soc., 3, (1996) 585-593.

[32] W.P.Thurston, Three dimensional manifolds, Kleinian groups and hyperbolic geometry, Bull.Amer.Math.Soc., 6, (1982), 357-381

[33] A. T. Vasquez, Flat Riemannian manifolds, J.Differential Geom. 4 (1970), 367-382

[34] R. Waldmüller, A flat manifold with no symmetries, Experiment. Math. 12 (2003), $71-77$

[35] J.Wolf, Spaces of constant curvature, McGraw-Hill, 1967.

Institute of Mathematics

University of Gdańsk

ul. Wita Stwosza 57

80-952 Gdańsk

Poland

E-mail: matas @ paula.univ.gda.pl 